\title{
Les personnages de la comédie ancienne et le théâtre français
} Marie Delcourt

\section{Citer ce document / Cite this document :}

Delcourt Marie. Les personnages de la comédie ancienne et le théâtre français. In: Bulletin de l'Association Guillaume Budé, $\mathrm{n}^{\circ} 1$, mars 1964. pp. 103-110;

doi : 10.3406/bude.1964.4060

http://www.persee.fr/doc/bude_0004-5527_1964_num_1_1_4060

Document généré le 30/05/2016 


\section{Les personnages de la comédiè ancienne et le théâtre français*}

La scène tragique donne la solidité du vivant à ses héros, 'quelque figure qu'ils aient eue avant elle dans la légende ou les récits épiques. Après quoi ils poursuivent leur vie propre grâce à ces malentendus féconds qui, dans chaque pays et à chaque époque, renouvellent leur signification ${ }^{1}$. Une telle autorité manque aux personnages de la comédie, qui représentent, sans plus, l'intersection d'un caractère et d'une situation. Lorsqu'il est question de théâtre comique, le mythe ne peut être que social ou moral.

Rotrou reprend trois sujets traités par Plaute. Il retouche à peine l'argument d'Amphitryon qui devient Les Sosies (joués en I636), mais il donne un semblant d'existence à Thessala, suivante d'Alcmène, et lui fait échanger quelques mots avec Sosie : Molière profitera de l'indication et placera le couple SosieCléanthis en face du couple des maîtres et, du reste, pour contraster avec lui.

Les prisonniers de guerre sont une pièce presque tragique. $\mathrm{Au}$ centre est un père auquel on a volé un fils tout enfant. Le hasard lui livre ce fils qu'il ne connaît pas et qu'il est sur le point de faire torturer, puis mourir. Le voleur était un esclave trop chéri, auquel le maître accordait une confiance excessive. La scène où le père et le mignon vieilli se retrouvent face à face est d'une âcreté que la pudibonderie des commentateurs a fort à faire d'atténuer. Rotrou fit jouer ses Captifs peu après Les Sosies. Il a supprimé l'épisode gênant : Hégion est le plus vertueux des vieillards. De plus, alors que la pièce latine n'a pas de rôle de femme, Rotrou en imagine trois : la fille d'Hégion, la fiancée de l'enfant volé et une suivante.

Dans Les Ménechmes latins, le quiproquo s'éternise parce que les deux jumeaux ont chacun intérêt à le faire durer. Rotrou leur prête à tous deux une honnêteté parfaite, fâcheuse pour la vraisemblance. De plus, la courtisane Erotie, maîtresse, dans l'original, du frère marié, devient une jeune veuve; le Ménechme célibataire s'éprend d'elle et l'épousera au dénouement.

Ces gauchissements sont significatifs. Le public français de I640 supporte mal l'impartialité de Plaute devant ses person-

\footnotetext{
* Communication présentée au $7^{\circ}$ congrès de l'association Guillaume Budé Aix-en-Provence ( $1-6$ avril 1963 ).

1. Cf. Aureliu WeIss, Le destin des grandes auvres dramatiques, 1962.
} 
nages. Il désire prendre parti et qu'on lui donne de bonnes raisons de le faire. Et il souhaite entendre parler d'amour. On comble ses vœux en lui montrant un jeune couple persécuté qui poursuit son bonheur en bernant ses tyrans. Les spectateurs se solidarisent avec les amoureux, applaudissent aux bons tours qu'un valet ingénieux joue au fâcheux, et, au dénouement, triomphent avec ceux qui incarnent leurs propres aspirations. Dans Le pédant joué (imprimé en 1654), il y a deux couples. La pièce contient des scènes d'un talent extraordinaire, avec seulement trop de morceaux traités en médaillons, sans regard à l'ensemble. Elle est toute marquée par le goût du temps pour les sujets romanesques où la sympathie penche toute d'un seul côté.

Assurément, cela n'était point neuf. Toutes les comédies de Térence sont sentimentales, sauf la dernière, Les Adelphes, qui se rapproche de la conception plautienne. L'auteur semble d'abord donner raison à Micion, père indulgent et complaisant. Mais à la fin le sévère Déméa prend sa revanche et oblige son frère, prisonnier de son personnage, à des concessions que l'autre trouve fort désagréables. Cela apparaît peu, parce que Térence est incapable de donner à aucun trait le grossissement qui le rendrait immédiatement sensible. De plus, les commentateurs, unanimement, faussent le sens de la pièce en l'interprétant à travers Molière. En I66r, Molière a tiré du contraste entre les deux frères le premier acte de L'école des maris (les deux derniers développent un thème de fabliau que Boccace a utilisé dans sa XXIII ${ }^{\circ}$ Nouvelle et Lope de Vega dans la Discreda Enamorada) où il donne totalement raison à Ariste, totalement tort à Sganarelle. Le Déméa de Térence, haute figure de gentleman-farmer, travailleur, avare, dur à lui-même autant qu'aux autres, au surplus insupportable, devient un tyranneau vaniteux bon à berner. L'école des femmes - qui n'a aucun modèle antique - est aussi une pièce romanesque, mais il y a en Arnolphe ce qui manque à Sganarelle, une passion vraie. Le spectateur se réjouit de le voir échouer, mais, s'il réfléchit, il sent en lui-même des désirs aussi déraisonnables, aussi irrépressibles que ceux d'Arnolphe et promis à la même défaite. C'est cette impartialité secrète, dissimulée, qui donne à la pièce sa valeur profondc.

Avant Térence, Ménandre son maître avait écrit des comédies sentimentales, où après quelques méprises, tout s'arrange pour le plus grand bonheur des gens de bien. Entre Ménandre et Térence, Plaute fait figure de solitaire lui qui ne croit guère aux gens de bien et qui leur accorde rarement un dénouement optimiste. 'C'est peut-être une des raisons pourquoi le xvire siècle a mis Térence si haut, le déclarant toujours supérieur à Plaute, alors que c'est chez Plaute que les auteurs vont chercher des 
sujets. Cyrano de Bergerac écrit une pièce sentimentale, mais c'est dans Les scours Bacchis qu'il a trouvé quelques-unes de ses meilleures inspirations : un type de pédant, un autre de valet astucieux, le thème du désir sénile, et une histoire de galère promise à une rare fortune sur la scène française.

Quant à Ménandre, le classicisme français en a fait son maître, alors qu'il n'en lisait que des fragments. Il y a là pour nous une énigme, et même deux. La première est que l'œuvre de Ménandre, une des plus populaires de l'Antiquité, ne soit pas arrivée jusqu'à nous. La seconde est la place qu'il tient dans l'esthétique de $\mathrm{xviI}^{\mathrm{e}}$ siècle, alors qu'on en connaissait fort peu de chose. Le début de la Renaissance prépare une sorte de mythe de Ménandre, en vertu de quoi les auteurs, de Montaigne à La Fontaine, parlent de lui comme s'ils le lisaient.

Mais à l'époque romaine, alors qu'on le lisait encore, circulaient déjà des collections de Monostiques. En I 553, Guillaume Morel, imprimeur parisien et humaniste, fait sortir de ses presses un recueil de fragments : Ex Comoediis Menandri quae supersunt, texte grec et traduction latine. Jacob Hertel (1560), Nicolas Rigault (1613), Hugo Grotius (1709) en donnèrent d'autres, qui eurent le même succès. Au surplus, bien avant cela, les studieux étaient retournés prendre aux sources les débris de l'œuvre où ils sont, dans le florilège de Stobée, le Banquet des Sophistes d'Athénée, les lexiques de Suidas et d'Etienne de Byzance. La Nationale possède la princeps d'Athé-

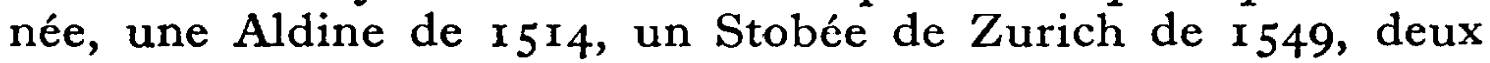
Suidas, l'un de Milan, de 1499, l'autre de Venise, de 1514, $^{14}$ tous reliés aux armes de Henri II. Voilà pour les citations textuelles. L'admirable est qu'avec ces morceaux épars soustendus par les jugements des anciens on ait pu faire de sa poétique une sorte d'image exemplaire et de lui un auteur vivant. Il est vrai qu'en I 853 Guillaume Guizot a écrit sur lui un ouvrage auquel il n'y a rien à reprendre et peu à ajouter, malgré les découvertes de 1906 et de 1959. Pour nous qui regardons les choses de l'extérieur, nous reconnaissons une certaine sagesse ménandrienne, chez Molière, dans les rôles de frères, ce Cléanthe, cet Ariste, ce Béralde, qui cherchent à ramener à la raison Orgon, Philaminte, Argan. Le raisonnable travaille toujours au bénéfice des amoureux, ce que Ménandre eût approuvé.

Le monde de Ménandre est dominé par quelques mythes que Plaute et Térence ont respectés. Ce n'est pas que la société romaine ne différât de la grecque; mais Rome précisément n'admettait sur les planches certaines réalités choquantes qu'à la condition qu'elles lui fussent présentées sous le vêtement 
hellénique. Si elle n'a pas eu de tragédies à sujets romains, c'est que les légendes grecques étaient riches et s'imposaient à un peuple doué de peu d'imagination fabulatrice. Si, dans le domaine de la comédie, il en fut à peu près de même, c'est en grande partie à cause des scrupules du cant romain. Dans une comédie grecque, c'est toujours un esclave qui combine et qui accomplit les fourberies. Il travaille au bénéfice de son jeune maître sans que celui-ci ait à se salir les doigts. Ainsi reste sauf le prestige de l'homme libre. En compensation, le jeune maître, éclipsé par son valet, fait figure d'imbécile, ce que Molière, dans L'étourdi, pousse à la limite. Berné par l'un et l'autre, le père est plus piteux encore. Le Romain s'en offensa et décida qu'un esclave ne pourrait avoir plus d'esprit que son maître, à moins que celui-ci ne fût grec. Au prestige de l'homme libre se superpose le prestige du Romain.

Molière a-t-il de même ménagé l'amour-propre de ses compatriotes ? On est tenté de répondre négativement parce qu'on pense à M. Jourdain. Et Sganarelle, L'école des maris, L'école des femmes, L'amour médecin se jouent à Paris. Mais est-ce un hasard si la scène de L'étourdi est à Messine, celle de Scapin à Naples, c'est-à-dire les deux pièces où les vieux messieurs sont le plus cruellement bafoués, au point que, pour la seconde, Boileau en marqua de la mauvaise humeur ? Dandin et Le Médecin malgré lui se passent à la campagne. Pourceaugnac et la comtesse d'Escarbagnas sont des provinciaux. Provinciaux et campagnards, à l'égard des Parisiens, sont des étrangers.

Le théâtre antique offrait au nôtre un vaste répertoire de valets trompeurs, modèles de nos Corbinelli et de nos Mascarilles, et, de plus, quelques esquisses de servantes astucieuses et sûres d'elles-mêmes, l'Astaphie, l'extraordinaire Pardalisca de Plaute, le couple Pythias et Dorias dans l'Eunuque. Pour mesurer ce que doivent à ces modèles la Lisette de $L$ 'amour médecin, la Dorine de Tartuffe, la Toinette du Malade, il faut comparer celles-ci à la Martine des Femmes Savantes, en qui Molière a stylisé, en dehors de toute influence antique, l'image qu'il se faisait du bon sens villageois. Quant au duo du valet ct de la soubrette qui tantôt fait écho à celui des maîtres (Dépit amoureux, Bourgeois gentilhomme, Dandin) tantôt lui sert de contrepoint (Amphitryon), Plaute lui en suggérait l'idée par une scène, unique en son genre, celle du Cordage où une idylle s'ébauche entre l'esclave du jeune premier et la servante de la jeune première. Cette pièce, inspirée de Diphile, est d'une sentimentalité toute ménandrienne. Il est permis de croire du reste que Molière n'aurait pas eu besoin de ce modèle pour imaginer le thème du valet amoureux réplique de son maître. 
Un bourgeois grec ou romain accepte à la rigueur que l'on mette sur la scène un de ses pareils trompé par un esclave. Mais il ne saurait admettre qu'on le représentât, même par personne interposée, trompé par sa femme. La dignité de l'époux est intangible. Lui s'accorde toutes les libertés - du moins chez Plaute - ne prenant la peine de dissimuler que si la femme a une fortune personnelle qui la rend redoutable. Elle lui jouera bien en revanche quelque mauvais tour; mais qu'elle accueille un amant, ou qu'un homme quelconque lui fasse la cour, cela est parfaitement impensable. Des philologues candides en ont tiré des conclusions flatteuses pour la vertu des dames romaines, alors que le tableau n'a rien à voir avec la réalité. Il résulte d'un tabou qui fut assez puissant pour interdire au monde antique tout entier de jamais jouer avec l'idée du cocuage.

Molière en joue, parce que tout le moyen-âge français en avait joué, mais lui aussi se heurte à un tabou : non plus la dignité du mari, que les fabliaux bafouent superbement, mais bien la pudibonderie du règne de Louis XIV. L'audace verbale de Cyrano de Bergerac suffirait à prouver que sa pièce date de la Fronde. Molière n'ose mettre en scène que des imaginations et des craintes : celles d'Arnolphe, de Sganarelle. Le Sganarelle du Mariage et George Dandin sont sûrs de leur affaire, mais c'est pour l'avenir. Au surplus, Dorimène ne se propose même pas de tromper son mari ; elle espère simplement qu'une prompte mort l'en débarrassera bientôt. Tout Don fuan parvient à tourner autour de la possession amoureuse sans jamais dépasser la simple galanterie, sauf en ce qui concerne Elvire qui appartient au passé de la pièce. Dans Le séducteur de Séville, tout est réalité et des plus concrète. Le parti-pris d'ignorer les choses sexuelles interdit aux amoureux de Racine de couronner leurs feux sans un mariage en bonne et due forme. Il atteint dans la comédie les maris aussi bien que les femmes. M. Jourdain ne trompe la sienne qu'en imagination ; le Sganarelle du Médecin se permet quelques privautés avec la belle nourrice Jacqueline : les choses ne vont pas plus loin. Inégaux devant le mythe antique, l'époux et l'épouse se retrouveront égaux devant le mythe créé par l'éthique française $\mathrm{du} \mathrm{XvII}{ }^{\mathrm{e}}$ siècle.

La loi athénienne ne reconnaissait de valeur légale qu'à l'union conclue avec une femme libre et citoyenne. A Rome, un mariage inégal reste dépourvu d'honesta celebratio. Les jeunes filles de bonne famille étaient si sévèrement cloîtrées qu'elles ne pouvaient paraître sur le théâtre. On y voit seulement soit quelques filles sans parents, pauvres et vertueuses, soit des courtisanes ou futures courtisanes exploitées par des tenanciers de maison de passe ou par leur propre mère. Lorsqu'un amou- 
sincère unit le jeune premier avec la déclassée, elle est immanr quablement reconnue libre, citoyenne et digne d'être épousée. Il en est de même de celle qui est promise à la prostitution, mais qui a su se garder pour un seul amant. Ménandre et Térence ont abondamment usé de la reconnaissance, Plaute beaucoup moins, et il l'expédie en quelques vers lorsqu'il ne peut dénouer autrement une action. Le mythe de la vertu récompensée est pour lui sans prestige. On imagine avec quel rire sardonique il lui arrive d'y sacrifier.

La claustration des filles dans l'Antiquité était interrompue par des fêtes religieuses qui souvent avaient lieu la nuit. Cela donne aux auteurs un ressort commode : dans le désordre d'une cérémonie nocturne, un garçon viole une fille et lui enlève un objet qui leur permettra de se retrouver; la fille met au monde un enfant; après bien des traverses, les deux partenaires se reconnaissent . Jamais un garçon n'hésite à épouser une femme qu'il a rendue mère. Il est peu probable que cette convention corresponde à la réalité, mais sa constance atteste le prix que les anciens attribuaient, non peut-être à la personne de l'enfant, mais à la certitude d'une postérité.

La scène française ramène cette bigarrure au cadre d'une famille schématique composée des parents (et plus souvent d'un père veuf), d'un ou deux enfants, d'un ou deux valets et l'un ou l'autre collatéral. La jeune fille apparaît sur le théâtre. Elle n'a aucune substance psychologique, n'existant guère que comme amoureuse, avec la permission de s'associer aux tromperies qui assurent sa libération. Les anciens considéraient une séduction comme plus choquante qu'un viol, lequel satisfaisait leur sadisme inconscient et leur idéal de passivité féminine. Les jeunes filles de Molière, même Agnès, sont déjà éprises et engagées lorsque la pièce commence, et rien ne les fera se dédire. En revanche, le public ne saurait supporter l'idée d'un lien quelconque entre amour ou mariage, d'une part, et naissance d'enfant de l'autre, - ni davantage le spectacle des jeunes courtisanes vierges encore ou déjà rompues aux astuces du métier, - des vieilles qui ne sont plus que des habilleuses ou des conseillères, - des mèrcs qui ne voient pour leur fille d'autre méticr que celui qui leur a permis de les élever. C'est justement dans ces peintures que l'impartialité de Plaute s'exerce avec le plus de génie. La suppression de ce milieu interlope a fait pencher la comédie française vers la sentimentalité, en enlevant à l'attendrissement les contrepoids qu'il a chez Plaute. Dans le Brave, où Baïf adapte L'officier fanfaron, la courtisane devient une petite bourgeoise appauvrie; Rotrou fait d'une autre une jeune veuve. Les La Tremblaye dans Le pédant joué 
sont des gens déchus, restés à peu près honnêtes. Molière essaie timidement de garder quelque chose de la variété sociale du milieu antique; dans Pourceaugnac, Nérine, une "femme d'intrigue ", et Sbrigani, "homme d'intrigue ", Napolitain ; dans le Bourgeois, deux aventuriers, Dorante et Dorimène, qui se repentent à temps; - dans L'avare et dans Scapin, deux déclassées semblables à celles que le théâtre antique osait mettre sur la scène. Hyacinthe, qui vit avec une mère adoptive, est l'exacte réplique de l'Antiphile du Bourreau de soi-même. La pièce commence qu'elle est déjà mariée avec Octave, ce qui permet à Molière d'escamoter les acheminements. Il ne pouvait en faire autant pour Mariane, objet de rivalité entre Harpagon et son fils. Le drame de Mariane est celui des courtisanes de Plaute, la pauvreté. Molière ne pouvait la montrer vendue à Harpagon par sa mère, qui en aurait été ou odieuse ou excessivement pitoyable. Il a chargé du maquignonnage Frosine, qui vient en droite ligne des maquerelles de Plaute, avec cette différence qu'elle ne travaille pas pour son propre compte, mais cherche à servir les amoureux.

Dans la comédie antique, la jeune fille, même si légalement elle peut épouser le garçon, est toujours d'un rang ou du moins d'une fortune inférieurs aux siens. Il l'élève jusqu'à lui. La fille assurément n'avait de droit strict à un héritage que si elle n'avait pas de frère; sa dot dépendait du bon vouloir de son père. Je pense cependant que cette obstination à montrer invariablement des filtes humiliées ne reflète pas tant une réalité sociale que cette même tendance inconsciente qui fit qu'on s'offusqua moins d'un viol que d'une séduction. L'image d'une fille livrée à un garçon paraissait plus satisfaisante que celle d'un homme obligé d'alléguer un sentiment pour avoir à sa merci celle qu'il désire.

Le tableau change dans un pays où les filles sont dotées, où, davantage, une tradition d'amour courtois et de galanterie a modifié, dans la littérature sinon dans la vie, les rapports entre les sexes. La question d'argent joue un rôle faible dans les mariages de Molière. Trissotin est un assez pauvre coureur de dot et l'héritage de Léandre n'est là que pour amener la réplique de Géronte : "Monsieur, votre vertu m'est tout à fait considérable et je vous donne ma fille avec la plus grande joie du monde " (Médecin, III, 9). Ce qui empêche les unions, ce n'est pas la disproportion des fortunes, c'est la lubie d'un obsédé. Seules, Hyacinthe et Mariane, pauvres et vertueuses, se trouvent dans la situation que Ménandre et Térence traitent avec prédilection. Octave raconte à Scapin sa première rencontre avec Hyacinthe : "Une autre aurait paru effroyable dans l'état où elle était, car elle n'avait pour habillement qu'une méchante 
petite jupe... ". Telle est exactement Phanium dans Phormion, Antiphile dans le Bourreau, avec une servante crasseuse pour rehausser le tableau, la saleté prouvant la misère et celle-ci la vertu. Ces abandonnées passant de la pire détresse au plus grand amour sont des héroünes de roman populaire. "Elle avait à pleurer une grâce touchante et sa douleur était la plus belle du monde ». Un mot de plus, et la comédie sentimentale devient comédie larmoyante. Mais l'interlocuteur d'Octave est Scapin, et si Octave est un personnage de Térence, Scapin, émule de Pseudolus, est un personnage de Plaute.

Lorsque Molière écrit $S$ capin, il a dépassé la comédie sentimentale. Cela ne veut pas dire qu'il renonce à faire entrer le public dans son jeu, ni à l'émouvoir — dans le Bourgeois, dans le Malade, - en faveur des amours contrariées. Mais, dans un autre registre, il a donné des chefs-d'œuvre du comique d'impartialité. Deux ans après L'école des femmes, où la sympathie penche d'un seul côté, Le mariagé forcé, œuvre courte, magistrale, est toute dans l'esprit de Plaute. Même absence de contrepoint sentimental dans George Dandin. Pourceaugnac, Escarbagnas, sont des farces plautiennes ainsi, que relevé d'un peu de romanesque, L'amour médecin. Mais c'est dans la plus haute de ses comédies que Molière devait atteindre le sommet de l'impartialité comique, en mettant face à face les deux êtres les moins faits pour se rendre heureux. Rien ne paraît plus loin de Plaute que Le misanthrope, et cependant Molière n'aurait pu écrire une telle ouvre s'il était resté prisonnier de la formule sentimentale. Celle-ci lui avait dicté le dénouement optimiste qui gâte Don Garcie. Dans Le misanthrope, il a préféré, comme Plaute l'eût fait, ne pas conclure.

L'avare doit à Plaute moins qu'il ne semble : le thème de la cassette dissimulée, le pathétique monologue du volé et un vers qui lui a fourni le nom d'Harpagon ${ }^{1}$. Tout le reste est de lui, et avant tout l'idée, tout à fait neuve, d'un vice qui fait tache d'huile et dégrade autour de lui les caractères, chacun à sa manière. Comme dans Le misanthrope, nous avons là une forme d'impartialité qui dépasse tout ce que Plaute a inventé, mais qui dérive de sa vision du monde.

Il faudrait suivre après Molière la dialectique du comique romanesque et du comique impartial, et décrire leur alliage dans le cynisme et la sensualité sèche du début du XvrrI ${ }^{\mathrm{e}}$ siècle. A quoi Les Ménechmes de Regnard, comparés à ceux de Rotrou, donneraient une bonne entrée en matière.

Marie DelcourT,

x. Aurum mihi intus harpagatum est (201). 